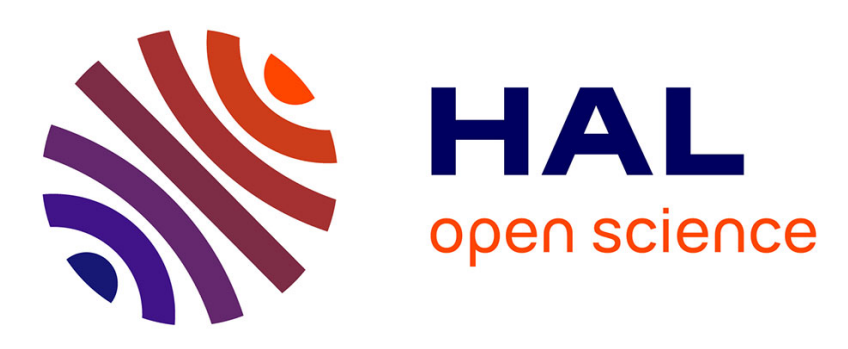

\title{
Formation des nouveaux espaces urbanisés et recomposition socio-spatiale des villes sahariennes du Sud-ouest
}

\author{
Badreddine Yousfi
}

\section{To cite this version:}

Badreddine Yousfi. Formation des nouveaux espaces urbanisés et recomposition socio-spatiale des villes sahariennes du Sud-ouest. Les mutations de la ville saharienne - Approches croisées sur le changement social et les pratiques urbaines, Faculté des Sciences Sociales et Humaines-Université Kasdi Merbah, Ouargla., Mar 2015, Ouargla, Algérie. halshs-01235679

\section{HAL Id: halshs-01235679 \\ https://shs.hal.science/halshs-01235679}

Submitted on 2 Dec 2015

HAL is a multi-disciplinary open access archive for the deposit and dissemination of scientific research documents, whether they are published or not. The documents may come from teaching and research institutions in France or abroad, or from public or private research centers.
L'archive ouverte pluridisciplinaire HAL, est destinée au dépôt et à la diffusion de documents scientifiques de niveau recherche, publiés ou non, émanant des établissements d'enseignement et de recherche français ou étrangers, des laboratoires publics ou privés. 


\section{Formation des nouveaux espaces urbanisés et recomposition socio- spatiale des villes sahariennes du Sud-ouest}

Yousfi Badreddine, Maître de Conférences, HDR, en géographie et aménagement du territoire, Université d'Oran II- Mohamed Ben Ahmed, Chercheur au Laboratoire LEGEAT

Les nouveaux espaces périphériques dans les agglomérations urbaines sahariennes sont le produit de succession d'actions qui viennent répondre aux besoins de la population en matière de logement et d'équipement. Ces actions, entreprises depuis la colonisation et qui se sont accélérées après l'indépendance ont façonné progressivement ces villes et leurs quartiers qui se distinguent tant structurellement que fonctionnellement.

Ce dynamisme se caractérise sur le plan spatial par un étalement considérable du cadre bâti, produisant des nouvelles périphéries urbaines aussi bien programmées que spontanées. Loin d'être homogène, cette ceinture d'habitat périphérique renferme des noyaux d'habitat qui se distinguent aussi bien par leur morphologie que par leur contenu social. En effet, les nouveaux programmes affectés dans ces espaces ciblent des groupes sociaux différents comme pour les logements de fonction, les logements socio-participatifs et les logements sociaux. Par ailleurs, la population issue des milieux défavorisés trouve des difficultés pour s'insérer dans ce système ; elle s'installe dans les marges des villes produisant des quartiers d'habitat précaire, non réglementaire.

Quelles sont les logiques et les stratégies d'insertion de ces nouveaux habitants dans les villes de Sud-ouest algérien ? Quelles sont les pratiques d'habitat qui ont été développés dans ces espaces?

\section{I- Des villages coloniaux face aux ksour : ouverture de l'espace oasien}

La première étape de la formation des villes sahariennes remonte à la période coloniale où des villages ont été créés à proximité des ksour les plus importants pour accueillir au départ des casernes militaires. Ceci a contribué à la constitution d'une nouvelle trame urbaine, normalisée selon une logique techniquo-militaire ${ }^{1}$ où les villages coloniaux font face aux ksour.

Cette période s'étale entre 1900 et 1930 et concerne un ensemble d'oasis chefs d'annexe ou postes militaires telles qu'Adrar, Aoulef, Reggane, Timimoun, Zaouiet-Debagh (dans l'actuelle wilaya d'Adrar) et Beni-Abbès, Taghit, Igli, Abadla, Beni-Ounif et Colomb-Bechar (dans l'actuelle wilaya de Béchar) situées aujourd'hui dans la wilaya de Bechar. Ces postes militaires, dont la fonction est d'instaurer l'ordre et de pacifier la région, étaient installés au départ à l'intérieur des remparts des ksour. Rapidement, les espaces situés aux alentours de

\footnotetext{
${ }^{1}$ Il faut rappeler que les territoires sahariens, étaient sous statut militaire depuis leur colonisation jusqu'à leur départementalisation en 1956. Jouissant d'une quasi-autonomie, l'intervention des officiers militaires du Bureau des Affaires indigènes dans l'espace était timide et consistait à assister la population locale par la construction des infirmeries, des écoles... Ces équipements élémentaires et ces installations militaires vont constituer les noyaux de villages qui vont bouleverser le mode d'habitat dans les espaces oasiens.
} 
ces ksour ont été occupés pour construire quelques petits équipements, mais aussi pour abriter des populations extérieures tant européenne qu'indigène, venues s'installer dans ces agglomérations.

Les nouveaux espaces extérieurs ont été pensés pour accueillir une population vivant selon le modèle européen. La ville telle qu'elle a été conçue par les nouveaux plans à partir de 1940 négligeait les indigènes et a dressé des barrières entre les deux espaces (Souami T., 2004). A Kenadsa, la mine a eu des effets non négligeables sur l'urbanisation où un quartier est réservé pour les européens et un autre a été aménagé pour les travailleurs musulmans². A Beni Ounif, le douar arabe, abritant une population appartenant aux différentes tribus de la région, s'est développé autour de la gare terminus temporaire sur la ligne de chemin de fer Oran- ColombBechar.

L'insertion économique de la population locale, qui s'est manifesté par l'avènement de l'emploi salarial dans les chantiers des hydrocarbures et de bâtiment à partir des années 1940, a produit des mobilités résidentielles en direction de nouveaux espaces extramuros. L'émancipation des harratine et la fixation des nomades ont accéléré ces mutations sociospatiales, contribuant à l'édification des nouvelles habitations à l'extérieure du ksar. L'exemple de Timimoun illustre bien ce processus comme cela avait été bien démontré par Bisson J. (1960). Beaucoup de harratine avaient quitté le ksar pour s'installer dans un nouveau quartier nègre dans le nouveau village ; parallèlement les Chaânbi ${ }^{3}$ (commerçants, transporteurs) avaient constitué les premiers quartiers indigènes ${ }^{4}$ dans le nouveau village. Quant à la population locale constituée en particulier de propriétaires terriens à savoir les chorfa et les merabtine dont la rente agricole est la seule source financière, elle reste fixée dans ses habitations dans le ksar comme à Timimoun et Adrar.

Ainsi, les nouveaux espaces urbanisés des agglomérations sahariennes ont été constitués de populations extérieures aux oasis et même au Sahara : les européens occupant les maisons intégrées dans le nouveau village, les travailleurs musulmans venus du nord s'installant dans le quartier des ouvriers (Kenadsa) et les ex. méharistes Chaânbi accompagnant les militaires de la colonisation venus du Sahara central s'installant dans les nouveaux villages indigènes à Adrar (le quartier Hattaba), à Timimoun et à Beni-Abbès.

\section{II- Naissance de quartiers d'habitat autoproduit : réactivation des réseaux tribaux et familiaux}

L'introduction des codes d'urbanisme pendant la colonisation qui voulait contrôler et normaliser techniquement les agglomérations sahariennes n'a pas empêché la création et le développement des quartiers irréguliers, autoproduits par la population locale. Ce phénomène s'est prolongé après l'indépendance donnant naissance à certains quartiers d'habitat insalubre

\footnotetext{
${ }^{2}$ La majorité des travailleurs musulmans résidants dans le quartier des ouvriers à Kenadsa est originaire de la Kabylie et en particulier de Jijel (Capot Rey R., 1953).

${ }^{3}$ Appartenant à la tribu nomade des Chaânba parcourant le Sahara central et le M'zab. Les Chaânbi méharistes, originaire de la région du M'zab ont profité de leur statut de la police du désert pour accéder à ces espaces et se convertir en commerçants et transporteur par excellence.

${ }^{4}$ Appelés G'raba (les gourbis) dans certaines villes du Tell oranais (Saida, Sidi Bel Abbès, Ain Temouchent...).
} 
en particulier à Adrar et Bechar. Ces espaces se sont formés pour répondre au besoin de logements d'une population extérieure de plus en plus nombreuse, venue s'installer dans ces villes. .

Ces espaces sont le produit aussi bien de la sédentarisation ${ }^{5}$ que de l'émigration transsaharienne. Béchar par exemple a accueilli nombre de nomades alors que Beni Ouskout à Adrar s'est constitué en grande partie de Touareg maliens.

A Bechar, des quartiers informels ont été formés par la population indigène, nomade et ksourienne à la quête du travail dans le chemin de fer, le bâtiment et la houillère. Le premier quartier est né autour de l'ancien ksar dès les années 1940, sur les rives de l'oued Bechar appelé Chaâba. La décennie suivante est marquée par la naissance d'un nouveau quartier près de la gare ferroviaire. Sur la rive est de l'oued de Bechar, le quartier Debdaba prend de l'ampleur suite à l'installation des nouveaux nomades de la tribu des Ouled-Djerir' ${ }^{6}$. Au sud et en discontinuité avec la ville, un nouveau noyau s'est formé à proximité de la nouvelle exploitation houillère, constituant le quartier de Bechar Djedid appelé aussi Bidon 2, où quelques familles issues de la tribu des Doui-Meniaâ ${ }^{7}$ avaient édifié leurs habitations loin du centre colonial. Accentué par les choix politiques de développement économique du pays (industrialisation et tertiarisation de l'économie nationale), ce phénomène s'est accéléré après l'indépendance. Les deux quartiers de Bechar (Bechar-Djedid et Debdaba) ont connu une évolution spectaculaire due à la sédentarisation des nomades dont l'insertion se fait par les mêmes logiques d'appartenance tribale, observées pendant la colonisation. Les autorités locales ont dressé des plans de restructuration de ces espaces qui n'ont pas pu être réalisés, mis à part la réalisation des réseaux d'alimentation en eau potable, d'électricité et de l'assainissement. Quelques équipement et habitations publiques ont été greffés ça et là pour

\footnotetext{
${ }^{5}$ L'administration coloniale voulant se substituer au pouvoir exercé par des nomades sur les territoires sahariens, avait recouru très tôt à leur contrôle. Le nomadisme se rétrécit pour devenir pastoralisme. La circulation des nomades pasteurs fut encadrée par la loi de 1927 nécessitant d'octroyer une autorisation pour se déplacer avec leurs troupeaux vers le Tell, poussant de plus en plus les tribus nomades à parcourir les pâturages sahariens qui se sont à leur tour rétrécis en fonction des intérêts coloniaux dans la partie méridionale (Hadeid M., 2006).

${ }^{6}$ Ouled Djerir : tribu nomade qui parcourait les pâturages du nord-est de Béchar dans la zone située entre Beni Ounif et Oued Zouzfana.

${ }^{7}$ Doui Meniâ: Une grande tribu qui parcourait les pâturages de l'Oued Guir allant de Tafilalet au Maroc jusqu'au bas de l'Oued Saoura dont la fixation commença par le développement des houillères de Kenadsa et passa par le développement d'une activité agricole dans le secteur d'Abadla pour s'achever par une fixation forcée en 1958. Capot-Rey R. constate en 1952, que deux faits ont perturbé la vie traditionnelle de cette tribu : « le premier est le développement pris par l'exploitation des houillères, d'abord localisé à Kenadsa ; pendant la guerre, un deuxième puits a été ouvert à Bechar, puis un troisième à Sfia, une trentaine de kilomètres au nord du Guir: la mine va au-devant des nomades[...] l'autre fait a été la création en 1947, d'un secteur d'amélioration rurale SAR, le premier dans le Sahara ». Sur les 3000 travailleurs dans la houillère de Bechar, 2 300 font partie de la tribu de Doui-Meniaâ, selon le même auteur. Le processus de sédentarisation s'achève en 1958 lorsqu'une grande partie de cette population (8 000 personnes) fut fixée par l'armée française sous un prétexte sécuritaire dû au déclenchement de la guerre de libération en Algérie. Soupçonnée d'appuyer logistiquement le FLN en contribuant à l'acheminement des armes, les Doui-Menaâ avaient été fixés principalement à Abadla, Bechar, Kenadsa et Ksi-Ksou (Atlas des départements sahariens, 1960) ; la deuxième partie de cette tribu fut fixée à Tafilalet au Maroc, suite à l'instauration des frontières bien avant cette date.
} 
démarquer ces espaces. Des opérations de relogement ont concerné quelques familles en situation de détresse à Debdaba Bechar-Djedid, relogées dans les nouvelles zones d'extension de la ville (Zone Bleu).

Quant au quartier Beni Ouskout à Adrar, qui littéralement signifie « construis et tais-toi' » à Adrar, il a été édifié clandestinement à la fin des années 1970, dans la périphérie de la ville sur la route de Bouda. Une enquête ${ }^{8}$ réalisée en 2009 montre que 34,5\% des chefs de ménages sont nés à l'étranger, majoritairement au Mali. Pour la population née en Algérie, il s'agit essentiellement des autres wilayas sahariennes (Adrar, Bechar, Tindouf et Tamanrasset). Il faut dire que cet espace formé au départ par des refugiés maliens, devient par la suite un quartier qui intercepte les immigrants nationaux sahariens et même des familles mal logées de la ville d'Adrar, car le loyer est accessible aux ménages aux ressources financières limitées. La plupart de ces habitants se sont installés entre 1977 et 1989 ; ce processus a continué entre 1990 et 2010 (45\%). Il est aussi un quartier où les réseaux de solidarité sont activés puisqu'on trouve beaucoup des voisins qui ont des liens de parenté. Ce quartier constitue le support des migrations des Africains vers l'Europe, où beaucoup y transitent, séjournent et parfois s'y installent.

Ce quartier est resté jusqu'à ces jours dans l'insalubrité et pose autant de problèmes pour les autorités locales qui sont devant un dilemme : intervenir dans ce quartier exprimerait la reconnaissance des droits de ses habitants, alors que de nombreux sont en situation de séjour illicite. Un plan de restructuration du quartier décidé en 2000 avait échoué, car il proposait de déloger des ménages qui ont refusé les indemnités jugées injustes et insuffisantes (50 000 et 100000 DA). En injectant un programme de 60 logements dans les limites de ce quartier (route de Bouda), les collectivités locales ont trouvé l'alibi pour le doter de quelques équipements (une école primaire et un collège) et des infrastructures (réseaux divers). Ces réseaux n'ont pas concerné tout le quartier ; le bitume ne touche que la couronne marquant les limites entre l'espace institutionnel et l'espace autoproduit; le passage du goudron au sable dans ce quartier est le symbole de cette ambiguité des pouvoirs publics.

\section{III- Les nouveaux espaces urbanisés programmés par l'Etat : affirmation de la mixité sociale}

Si la priorité était donnée à la construction des équipements et des infrastructures dans les agglomérations sahariennes durant les deux premières décennies après l'indépendance (entre 1962 et 1980), les préoccupations des pouvoirs politiques se sont réorientées entre 1980 et 2010 sur la question du logement. De fait, la croissance spatiale des agglomérations sahariennes dans le Sud-ouest algérien, prend une dimension spectaculaire suite à l'instauration d'une série de mesures renforçant la production du logement dans les villes algériennes. Les lotissements, les coopératives immobilières et les zones d'habitat urbain nouvelles (ZHUN) sont à l'origine de ces extensions spatiales opérées entre 1980 et 1990, suivi d'un important programme entre 1990 et 2010 de logements combinant différentes formules injectées dans ces périphéries, à savoir l'habitat évolutif, social, socio-participatif...

\footnotetext{
8 (Baâla Z. et Ben Salem K., 2009).
} 


\section{IV-1 Introduction des logements de masse : logement public et système de quotas}

Bechar et Adrar, chefs-lieux de wilaya, ont bénéficié d'un programme important de logements. Après avoir été écarté de l'intervention des pouvoirs publics dans les années 1970, la ville de Bechar a été dotée d'une zone d'habitat urbain nouvelle Z.H.U.N de 1500 logements, qui n'a été réalisée que partiellement dans la fin des années 1980 à l'ouest de la ville.

A Adrar, la construction de quatre cités de logements collectifs a produit les premières poussées d'urbanisation dans la direction sud de la ville où viennent se greffer 837 logements dont une grande partie a été réservée pour les logements de fonction. Ces logements prennent la forme d'un habitat semi-collectif ne dépassant pas deux niveaux.

A cette période, le concept d'habitat social n'était pas encore clair, puisque cette forme d'habitat était ouverte à toutes les catégories sociales (cadres, employés, ouvriers...). Mais depuis la Loi de la cession des biens de l'Etat en 1981, ils ont connu une mutation de leur composante humaine due à une grande mobilité résidentielle.

Abadla, depuis sa promotion en chef-lieu de daïra, avait rencontré de nombreuses difficultés face aux besoins de ses habitants aussi bien en équipements qu'en logements. Après l'échec de la réhabilitation de l'ancien ksar, les autorités locales se sont orientées vers la construction d'une nouvelle ville sur l'ancien site militaire abritant une piste aérienne. Bénéficiant d'un programme spécial, d'une enveloppe budgétaire de 500 logements en 1976, l'étude et la réalisation de ce projet se sont heurtées à de nombreux conflits générés entre le Ministère, la wilaya et la commune (Hamidi A., 2003). Devant le retard dans la réalisation des équipements comme l'a signalé le même auteur (collège en 1979, lycée en 1989, hôpital en 1987), conjugué à l'insalubrité de l'ancien ksar, beaucoup de familles quittent Abdala pour s'installer dans les villages agricoles socialistes (villages de la révolution agraire) construits dans le cadre du périmètre irrigué de la plaine de Guir. Le volume de sa population a ainsi chuté pour passer de 9600 à 8200 habitants avec un taux d'accroissement annuel de $(-1.5 \%)$ entre 1977 et 1987. Le programme a été presque achevé dans les années 1990, avec la réalisation d'une zone d'équipement sur le bord de la route RN6 et une zone d'habitat semicollectif à l'intérieur. Issus d'un milieu nomade, les habitants s'adaptent mal avec ces nouveaux logements et conservent certaines pratiques rurales. L'élevage est pratiqué en plein milieu urbain et les toitures des logements collectifs sont aménagées en zriba (espace des animaux dans l'habitation traditionnelle) pour accueillir les animaux (voir photo 1). 


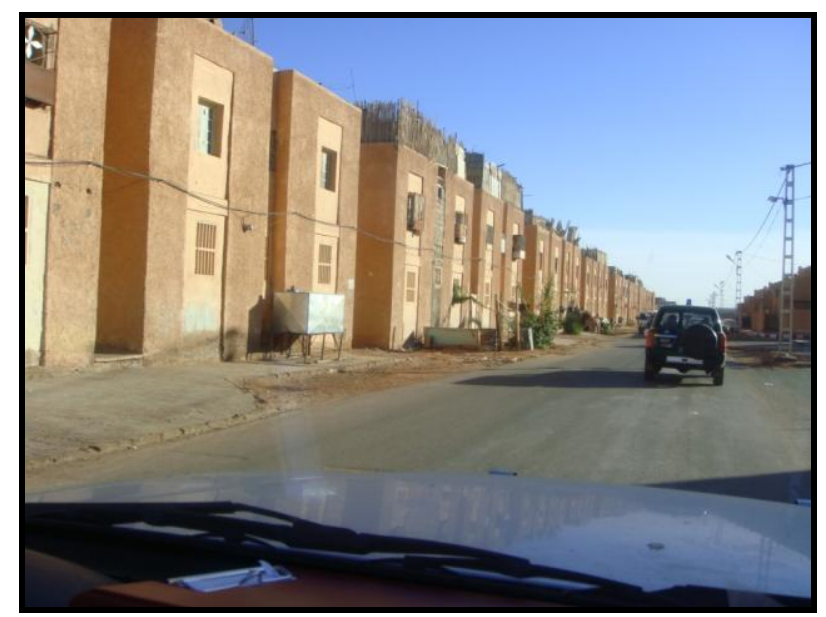

Photo n ${ }^{\circ}$ 1. La nouvelle Abbadla : pratiques d'élevage et d'engraissage sur les toits de bâtiments

\section{IV-2 Multiplication des formules de logements affectés dans les mêmes espaces à partir des années 1990}

De nouveaux programmes d'habitat ont renforcé la production du logement à partir des années 1990, visant différentes catégories sociales. Affectés par le biais des POS, ces quartiers planifiés se caractérisent par une mixité sociale due à l'injection de différents programmes d'habitat dans le même espace: logements sociaux, logements socioparticipatifs, logements évolutifs... Par ailleurs, la promotion immobilière est restée limitée pour ne pas dire inexistante, à l'exception de quelques opérations à Adrar et Bechar par le biais de la Caisse Nationale de l'Epargne et de la Prévoyance (CNEP). Dans les deux dernières décennies, le logement rural occupe une place importante et s'intègre dans le cadre de la politique de développement rural ; il a pour objectif la promotion des espaces ruraux et la fixation des populations locales. Mais un nombre important de ces opérations ont été réalisé dans l'espace urbain.

Des nouveaux quartiers sont nés par juxtaposition de ces différents programmes, notamment dans les villes de Timimoun, d'Adrar (nouvelles villes de Cheikh Mohammed Belkebir et

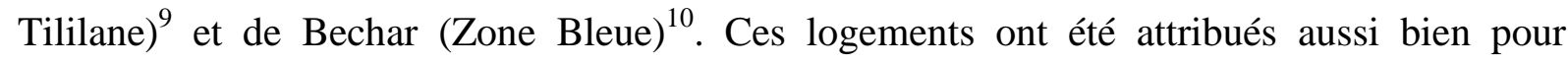
répondre aux malaises du logement de la population locale qui vit dans des conditions précaires d'habitat que pour loger en partie des nouveaux habitants installés dans la ville.

C'est ainsi, que la nouvelle composante humaine des nouveaux quartiers diffère selon le type des opérations de logement menées par le pouvoir dans ces espaces. En effet, si on prend l'exemple des la Zone Bleue à Bechar, située dans la ZHUN qui se caractérise par une

\footnotetext{
${ }^{9}$ Ville nouvelle est une appellation utilisée par les politiques en Algérie pour désigner les nouvelles zones urbanisées dans les périphéries urbaines. Il ne s'agit guère de la création de nouvelles villes proprement dite.

${ }^{10}$ Il s'agit de la Zone d'Habitat Urbaine Nouvelle (ZHUN) dont la réalisation a pris beaucoup de retard depuis son lancement et n'a jamais été complètement achevée. L'arrivée de nouveaux instruments d'urbanisme à partir de 1990, va permettre d'affecter de nouveaux programmes d'habitat dans cette zone par le biais du POS. Ainsi, l'appellation "' Zone Bleue'" est tirée depuis le POS de cette zone où la couleur bleue a été utilisée sur le plan pour tracer ses limites.
} 
dominance des logements sociaux et évolutifs, on constate que les origines géographiques des chefs de ménage sont reliées surtout à la ville de Bechar car ils représentent $(57,3 \%)$ de l'ensemble de la population (Abdelkader A.- Tlidji N., 2004). L'autre partie des habitants provient des autres communes de la wilaya de Bechar avec une dominance de Beni-Ounif, et des wilayas du Nord-ouest en particulier Oran et Naâma. Les mobilités intra-urbaines sont en rapport avec les conditions précaires d'habitat dans les anciens quartiers de ces habitants, notamment Zrikat et Debdaba.

Dans les nouvelles extensions de la ville d'Adrar où se concentrent les opérations du LSP ; seuls $14,2 \%$ des habitants sont originaires de la ville. Ainsi, les quartiers de Tililane et de Cheikh-Mohamed-Ben-Lekbir sont constitués d'une population provenant majoritairement de trois zones géographiques à savoir, les communes de la wilaya d'Adrar (Aoulef et Timimoun), les wilayas sahariennes (Bechar) et les wilayas du Nord-ouest (Oran). Ces mobilités sont en rapport avec l'emploi (embauche, mutation et promotion) représentant $60 \%$, et seulement $15 \%$ sont en rapport avec les conditions de vie et d'habitat. En effet les ksouriens s'orientent davantage vers la ville mais sans quitter leur lieu d'origine, ils investissent dans des résidences secondaires dans la ville d'Adrar, pour assurer des besoins résidentiels futurs de leur famille (travail, étude...). C'est ainsi qu'un nombre important des logements reste vacant dans les nouveaux quartiers planifiés à Tililane et Cheikh-Mohammed-Ben-Lekbir dans la ville d'Adrar.

Dans les autres villes de rang intermédiaire comme Timimoun, la population des nouvelles extensions formées par diverses opérations d'habitat, est majoritairement extérieure à la ville (Bouhafse S.-Ghenim, M. 2004). En effet, seulement 23\% des chefs de ménage des nouvelles extensions, sont originaires de l'agglomération (ville et ksour), contre 35\% pour les communes de la wilaya d'Adrar en particulier de la commune de Charouine et de Tinerkouk, $17,3 \%$ pour les wilayas sahariennes (Bechar et Ghardaïa) et $10 \%$ pour les wilayas du Nordouest. Ces mobilités sont liées aussi bien aux conditions de vie et d'habitat (38\%) qu'au travail (33\%). Le premier motif concerne les ménages originaires des communes limitrophes où la disponibilité et la qualité des services publics sont limitées ; quant au deuxième motif, il concerne les chefs de ménage extérieurs de la wilaya en particulier ceux venus du Nord.

Tableau ${ }^{\circ} 1$. Origine géographique des chefs de ménage des nouveaux espaces urbanisés selon leurs lieux de naissance : cas d'Adrar et de Bechar

\begin{tabular}{|l|c|c|l|c|}
\hline & $\begin{array}{l}\text { Zone Bleue } \\
\text { (Bechar) }\end{array}$ & Taux & $\begin{array}{l}\text { Tililane } \\
\text { et Cheikh-Med-Ben-Lekbir } \\
\text { (Adrar) }\end{array}$ & $\%$ \\
\hline Ville même & 176 & 57,3 & 12 & 14,2 \\
\hline Communes de la wilaya & 88 & 28,6 & 25 & 29,5 \\
\hline Wilayas sahariennes & & 0,6 & 21 & 24,7 \\
\hline Wilayas du Nord-ouest & 36 & 11,7 & 20 & 23,5 \\
\hline Wilayas du Nord-centre & 9 & 2,9 & 2 & 2,3 \\
\hline Wilayas du Nord-est & 5 & 1,6 & 2 & 2,3 \\
\hline Ensemble & 307 & 100 & 85 & 100 \\
\hline
\end{tabular}


Tableau $\mathrm{n}^{\circ}$ 2. Catégories socioprofessionnelles des chefs de ménage des nouveaux espaces urbanisés : Bechar et Adrar

\begin{tabular}{|l|c|c|c|c|}
\hline Catégorie Socio-Professionnelle & $\begin{array}{c}\text { Zone Bleue } \\
\text { (Bechar) }\end{array}$ & Taux & $\begin{array}{c}\text { Tililane et } \\
\text { Cheikh-Med-Ben-Lekbir } \\
\text { (Adrar) }\end{array}$ & Taux \\
\hline Commerçants et artisans & 27 & 9,6 & 07 & 8,7 \\
\hline Cadres supérieurs & 38 & 13,4 & 10 & 12,5 \\
\hline Cadres moyens & 114 & 40,1 & 50 & 62,5 \\
\hline Ouvriers qualifiés & 12 & 4,2 & 8 & 10 \\
\hline Employés et ouvriers non qualifiés & 93 & 32,7 & 5 & 6,3 \\
\hline Total 1 & 284 & 100 & 80 & 100 \\
\hline Sans emplois & 23 & 7,4 & 5 & 5,8 \\
\hline Total 2 & 307 & 100 & 85 & 100 \\
\hline
\end{tabular}

- $\quad$ Source : Foundou A., 2005, Abdelkader A.- Tlidji N., 2004

Dans les petits centres comme Reggane, les nouvelles extensions sont habitées par une population aussi bien locale qu'extérieure ; $46,1 \%$ des chefs de ménage sont originaires des ksour de la commune, contre 34,6\% pour les communes limitrophes en particulier de BordjBadji-Mokhtar (18\%), d'Aoulef et de Sali (Ibrahim A., 2010). L'emploi dans ce centre constitue le principal motif de ces mobilités (56\%). Dans cette localité, les chefs de ménage originaires des wilayas du Nord et de l'Ouest représentent 14\%. A Kenadsa dans les nouveaux quartiers, (lotissements et habitat social), la population est majoritairement originaire de la ville-mère (Bechar) ; 60\% y sont nés (Berbaoui A., 2000). Ceci s'explique par la faiblesse de l'attractivité de cette ville et sa dépendance totale vis-à-vis de Bechar : 60\% des chefs de ménage y travaillent. Kenadsa peut être qualifiée de "ville-dortoir".

Les catégories socioprofessionnelles des chefs de ménage des nouvelles extensions varient d'une ville à l'autre. A Adrar, elles sont composées principalement des agents de la fonction publique. Dans la Zone Bleue, à Bechar, les employés et les ouvriers non qualifiés émergent, reflétant une forte articulation entre les catégories socioprofessionnelles et la nature des opérations d'habitat dans ces espaces. En effet, les opérations LSP dans les quartiers de Tililane et de Cheikh-Mohamed-Ben-Lekbir, sont destinées à une catégorie ayant des sources financières moyennes, comme les agents de la fonction publique. Ces opérations permettent à ces nouveaux habitants d'accéder à la propriété de ces logements avec l'aide financière de 1'Etat via la Caisse National de Logement (CNL). Cependant, l'existence d'un nombre important de logements sociaux dans les la Zone Bleue à Bechar dont une partie a été réservée 
aux opérations de relogement, a contribué à l'installation d'une population limitée financièrement (employés et ouvriers non qualifiés).

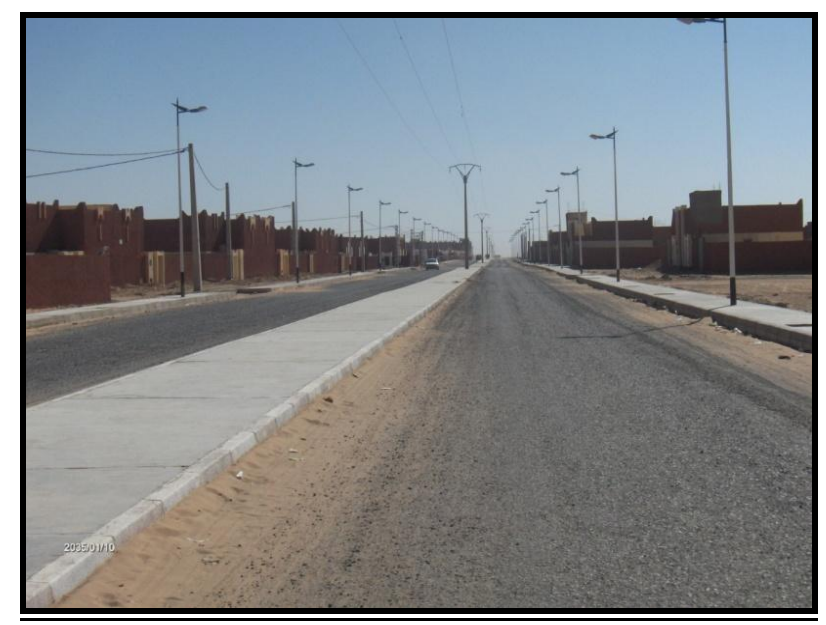

Photo n 2. Nouvelles extensions de la ville d'Adrar (logements LSP à Tililane)

Paradoxalement, une part importante des logements réalisés dans les communes urbaines entre 1990 et 2000 est constituée des logements ruraux (54\%). Affectés pour la promotion de l'habitat dans les zones des ksour et des palmeraies, ces programmes sont indirectement réinjectés au niveau local dans les zones urbaines afin de renforcer le volume des logements. Autrement dit, les autorités locales utilisent cette forme d'habitat planifié pour obtenir des financements auprès des pouvoirs centraux dans le cadre d'un programme national de l'habitat rural. Ensuite, ces financements sont affectés spatialement dans les zones de contacts entres les espaces urbains et les espaces ruraux, généralement près des ksour urbanisés dans les zones limitrophes des agglomérations. S'il est normal que la part des logements ruraux soit importante dans les communes semi-rurales ou rurales de la wilaya d'Adrar (Tinerkouk, Aougrout et Zaouiet-Kounta), elle est surprenante dans les communes urbaines où les pratiques de contournement des programmes d'habitat rural au profit des zones urbaines se sont succédées durant plusieurs années (44\% pour Adrar et Reggan plus $60 \%$ pour Timimoun et Aoulef). L'aide financière apportée aux populations dans ce cadre a contribué largement à la forte demande de ce dispositif. A Kenadsa, des tensions ont été enregistrées entre des citoyens et l'administration qui est accusée de manque de transparence suite à l'affectation d'un quota de logements jugé insuffisant (El Khabar de 18/03/2012).

\section{V-Intérêt particulier accordé aux logements des fonctionnaires : mécanisme sélectif} Dans la période des années 1970 et 1980, les nouveaux logements réalisés par l'OPGI, le maitre d'œuvre, n'ont pas un statut clairement prédéfini puisque des quotas sont attribués aux différents secteurs économiques (enseignement, santé, administrations,...). Ces quotas se négocient localement et résultent des rapports de force entre différents groupes sociaux et 
acteurs politiques. Ainsi, dans la même cité se côtoient différentes catégories socioprofessionnelles. Durant cette période, les logements de fonction ont eu une part non négligeable dans la ville de Timimoun par exemple, avec la construction des petites cités de 40 logements en 1983 affectés pour les secteurs de la santé, 30 logements pour l'enseignement et 50 pour les domaines de l'Etat en 1993.

Les logements de fonction constituent une forme importante dans la production du logement dans les agglomérations aussi bien urbaines que semi-rurales dans le Sud-ouest, soutenant la politique d'aménagement du territoire des zones sahariennes. L'ampleur de ces programmes varie d'un centre à l'autre et dépend de la concentration des services publics et du poids de chaque agglomération. Il est à signaler que ces logements sont construits sur un seul niveau donnant un aspect horizontal à ces nouvelles extensions qui se greffent dans la partie sud-est de la ville.

Situés sur les grands axes et bien desservies par les transports, les cités des fonctionnaires sont souvent bien aménagées, entourées de murs de clôture et parfois gardiennées comme la nouvelle cité des cadres des Directions exécutives, réalisée dans la partie nord à Bechar (photo 3). Plusieurs logements ont été concédés dans le cadre de la Loi de cession des biens de l'Etat de 1981 au profit de leurs occupants. Parmi les objectifs d'une telle action, il s'agit d'encourager ces cadres souvent non originaires de la région à s'ancrer dans la ville. Mais souvent ces logements sont revendus dans une stratégie de retour vers le Nord.

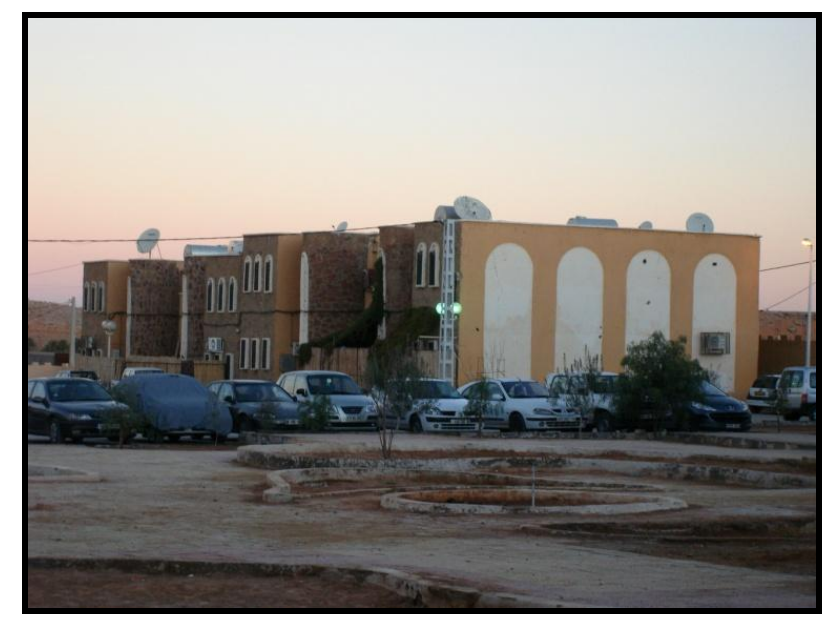

Photo $\mathbf{n}^{\circ}$ 3. Cité des fonctionnaires administratifs (Directions exécutives) à Bechar

La deuxième forme la plus répandue pour le logement des cadres passe par les coopératives immobilières. Instaurées en $1976^{11}$, ces coopératives ont connu un certain succès auprès des cadres supérieurs et moyens du secteur public. "Mieux informés, car proches de la source d'information, les gestionnaires du secteur public et ceux qui gravitent autour du pouvoir ont su mettre à profit le discours politique qui invoquait «les nouveaux besoins de la population " pour se "'placer" et ajouter sur les listes établies quelques membres de la famille élargie et ...quelques connaissances. » (Benjelid A., 1997, 5-26). C'est ainsi que ces opérations se

\footnotetext{
${ }^{11}$ Ordonnance 76-92 du 23 Octobre 1976 relative à l'organisation de la coopérative immobilière
} 
concentrent uniquement dans les villes les plus importantes à savoir Bechar, Adrar et Timimoun car leur création nécessite un montage bien particulier. Elles permettent à leurs bénéficiaires, d'acquérir des lots de terrain à des prix peu élevés.

Dans la wilaya d'Adrar, les coopératives immobilières ont constitué un moyen pour accéder à la propriété foncière. La démarche collective de construction des habitations n'a jamais été respectée et les coopératives ont pris l'aspect de lotissement comme cela avait été soulevé par Bendjelid A. (1997) en étudiant le cas de la métropole oranaise où « pour beaucoup, l'accès du marché foncier par le biais de la coopérative immobilière était une première étape vers l'appropriation privée $d u$ sol urbain bien plus intéressante que les avantages qui accompagnent cette opération. En ce sens, ce n'est pas tant la construction individuelle qui importait, mais l'acte juridique qui permettait de s'approprier à moyen terme une partie de l'assiette foncière, même si à l'échelon central, on voulait des maisons individuelles construites par des classes moyennes et non pour des classes moyennes du secteur public et même d'un secteur privé ( acteurs de la vie économique et culturelle...) de plus en plus (toléré», (Bendjelid A., 1997, p. 26).

A Adrar et à Timimoun les coopératives prennent une dimension considérable avec 29 coopératives immobilières constituant 1723 parcelles pour la première et 24 coopératives immobilières formant 1611 parcelles pour la deuxième, soit une moyenne de 60 parcelles par coopérative.

Ceci révèle la place qu'occupent localement certains groupes sociaux qui exercent des pressions pour s'octroyer le foncier dans ces deux villes et notamment les cadres du service public : coopératives de la santé, des services agricoles, de la justice, des affaires religieuses, de la sûreté nationale..., en plus de quelques coopératives du secteur économique représentées par quelques entreprises comme SONELGAZ et quelques banques publiques comme la BADR.

Majoritairement originaires des villes du Nord algérien et logés dans les logements de fonction, les bénéficiaires de ces coopératives ne s'engagent que rarement dans les opérations de construction, et préfèrent «stocker ce bien immobilier» pour les revendre quand ils se feront réaffecter dans les villes du nord ${ }^{12}$. Ceci explique bien l'état inachevé de ces lotissements formant des terrains immenses non construits dans les nouvelles périphéries, mise à part quelques habitations érigées çà et là. A Adrar, ces opérations ont bien marqué la nouvelle zone d'extension nord-ouest de Cheikh-Mohamed-Ben-Lekbir, donnant naissance à un quartier constitué par plusieurs petits ensembles d'habitat éclatés (voir et figure $\mathrm{n}^{\circ} 2$ photo $\mathrm{n}^{\circ} 4$ ). A Timimoun, la zone sud-est de la ville a les mêmes caractéristiques que celle d'Adrar. Ceci pose des problèmes de gestion pour les autorités locales qui n'arrivent pas à programmer des actions dans ces espaces, ralentissant les travaux d'aménagement des cités voisines formées par la réalisation des logements sous d'autres formes (LSP, social, évolutif), au moment où les travaux de construction dans les coopératives n'ont pas commencé dans la plupart des cas.

\footnotetext{
${ }^{12}$ La rotation de cadres est importante dans ces villes, puisque un nombre important ne passent que quelques années au sud dans une logique de gestion des carrières.
} 


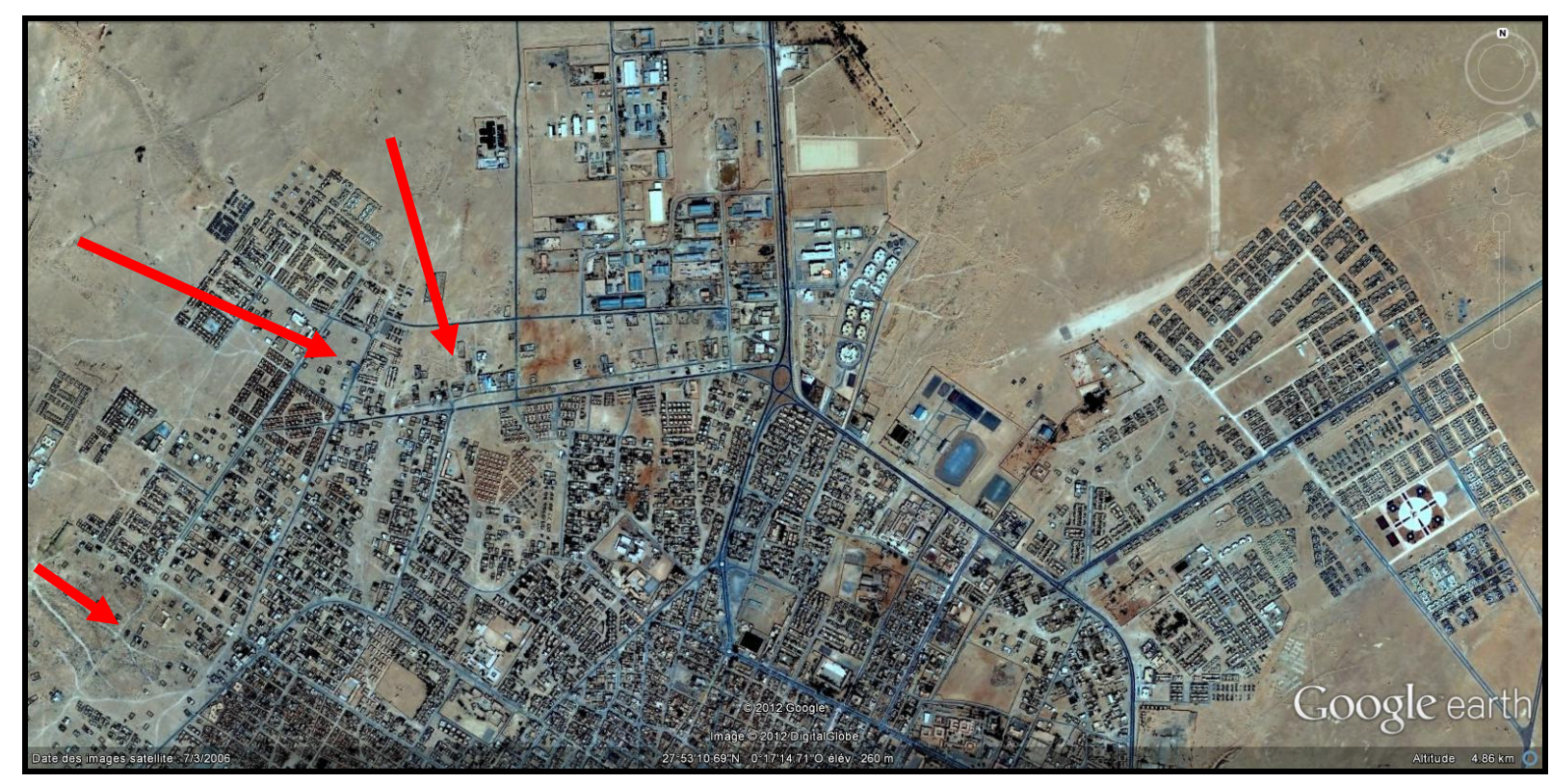

Figure ${ }^{\circ} 2$. Nouvelles extensions d'Adrar : ruptures dans le tissu urbain causées par le retard des réalisations des coopératives immobilières et des lotissements (partie nord-ouest)

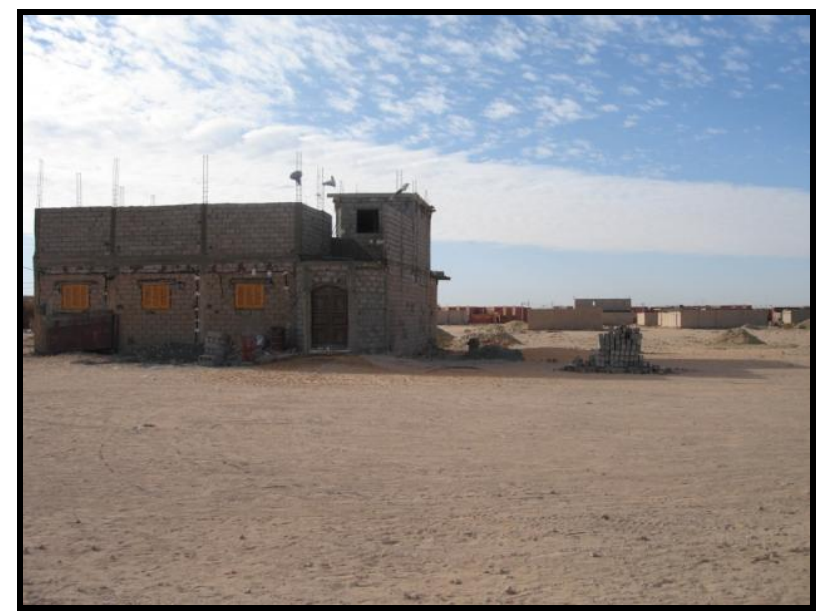

Photo ${ }^{\circ}$ 4. Coopérative immobilière à Adrar (Cheik-Mohamed-Ben-Lekbir) : retard flagrant dans la construction (excepté une salle de prière destinée pour la cité des logements sociaux et LSP)

\section{Reconquête du ksar et des jardins : naissance d'une forme d'habitat prestigieux}

Cette dynamique urbaine a eu des effets négatifs sur les anciens noyaux de ces villes à savoir les ksour qui se vident peu à peu de leurs habitants et tombent en ruine. Cette tendance s'est accélérée après l'indépendance où la population autochtone fuit peu à peu les anciennes habitations vers de nouvelles constructions dans les nouveaux espaces urbanisés. Ceci concerne la majorité des anciens ksour dans la Saoura qui sont abandonnés comme à Taghit, Igli, Beni-Abbès... 
Cependant, une tendance d'un retour aux ksour $^{13}$ et à leurs jardins est entrain de se former dans quelques oasis à tradition touristique dans le Sud-ouest algérien. L'afflux de nouveaux propriétaires avait été observé dans les jardins de l'oasis de Timimoun, qui a tendance à changer de vocation suite à la récession de l'activité agricole et à l'urbanisation. Achetés à des prix dérisoires (400 000 DA pour une parcelle d'un hectare en 2008), quelques Algérois ont pris l'initiative de construire une habitation avec jardin dans cette partie de l'oasis ${ }^{14}$. Ces habitations sont utilisées comme des résidences secondaires dans la plupart des cas, mais quelques unes ont un usage touristique (accueil d'hôtes, restauration, piscine et jardin) ${ }^{15}$. Le même phénomène est observé dans les palmeraies des oasis d'Adrar ville (Oucen Adgha, Ouled Ali), où les autochtones ${ }^{16}$, anciens résidents des ksour, réalisent des nouvelles habitations dans les jardins ${ }^{17}$.

A Taghit, une oasis touristique dans la wilaya de Bechar, le ksar avait été au centre de quelques opérations de restauration lancées dans la cadre du projet de la Route des Ksour ${ }^{18}$ en 2005. Cette opération est financée en grande partie par le Programme des Nations Unies pour le Développement PNUD, impliquant le Ministère de la Culture, les autorités locales, les associations et les habitants appelés directement à s'engager dans cette action. Certes cette opération a permis de restaurer le ksar devenu musée à ciel ouvert, abandonné par ses habitants. Exposé aux intempéries, les constructions, bien que 'retapées", se détériorent rapidement et l'activité touristique est très conjoncturelle, se limitant à un afflux de touristes nationaux pendant les fêtes de fin d'année et du mawlid (naissance du prophète). Dans cette perspective, comme à Timmimoun, quelques «nationaux nordistes » (expression qui désigne les Algériens originaires du nord de l'Algérie) ont pris l'initiative d'acheter des habitations

13 Observé au Maroc, les étrangers de nationalités française, allemande, hollandaise, américaine et espagnole
viennent s'installer dans les médinas et les vieux ksour, comme à Marrakech, retapant les maisons et aménageant
des Riadh. Signe de richesse, l'appropriation d'un bien immobilier dans ces espaces concerne aussi bien les
retraités des pays développés que des artistes, des cadres... Bien plus, ce phénomène a suscité l'intérêt de la
classe aisée marocaine des autres villes, affluant dans ces espaces afin d'acquérir une résidence secondaire. Si
une partie des habitations garde la même fonction, d'autres sont transformées pour des activités touristiques
(hébergement de touristes, restauration, cafés...). Ainsi, ces espaces se mondialisent, acquièrent de nouvelles
activités, valorisant ce patrimoine et provoquant de nouveaux intérêts, (KURZAC-SOUALI A.C., 2009).

${ }^{14}$ Nous en avons recensé au moins cinq durant notre visite de terrain effectuée en 2008, confirmée par les témoignages d'un habitant autochtone.

${ }^{15}$ En sachant bien que ces terrains sont non urbanisables ou "protégés", comment ces constructions ont vu le jour dans ces espaces et quelle a été l'attitude des autorités locales? La rumeur évoque l'intervention officieuse d'un haut responsable depuis Alger pour demander à l'administration locale de tolérer ces pratiques concernant quelques constructions.

${ }^{16}$ Il s'agit des propriétaires fonciers à savoir les chorfas et les merabtine qui ont construits leurs nouvelles habitations sur leurs parcelles agricoles. Il faut rappeler que le déclin agricole de ces vielles palmeraies et les mutations sociales qui ont affecté la société oasienne, notamment avec la tertiarisation de l'économie et l'urbanisation ont favorisé ces pratiques.

${ }^{17}$ Ces pratiques sont bien présentes dans les oasis les plus urbanisées au Bas Sahara et au M'zab.

${ }^{18}$ Cette opération cible les ksour de Taghit, Mogheul et Beni-Abbès dans la wilaya de Bechar ; les ksour de Timimoun, d'Akhled, de Charouine, Guentour, et Tinerkouk dans la wilaya d'Adrar ; les ksour de Ghardaïa, El Atteuf, la maison d'El-Atteuf dans la wilaya de Ghardaïa et le ksar du Témacine à Ouargla. Les ministères de la Construction, de l'Aménagement du Territoire et de l'environnement, de l'Intérieur, de l'Agriculture et du Développement rural sont invités à coopérer dans ce projet. 
dans le ksar, de les restaurer et de les réaménager pour accueillir des touristes ${ }^{19}$. Une association qui s'intéresse à la formation des jeunes et des femmes pour les métiers artisanaux (poterie, tissage...) s'est également installée dans le ksar avec comme objectif de le revaloriser sur le plan fonctionnel.

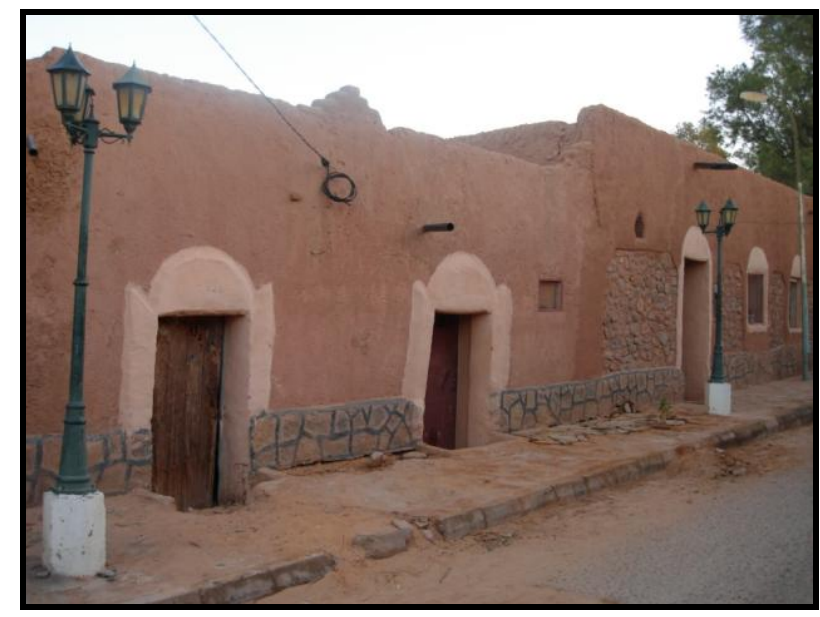

Photo $\mathbf{n}^{\circ}$ 5. Maison restaurée dans le ksar de Taghit (nouveau propriétaire algérois)

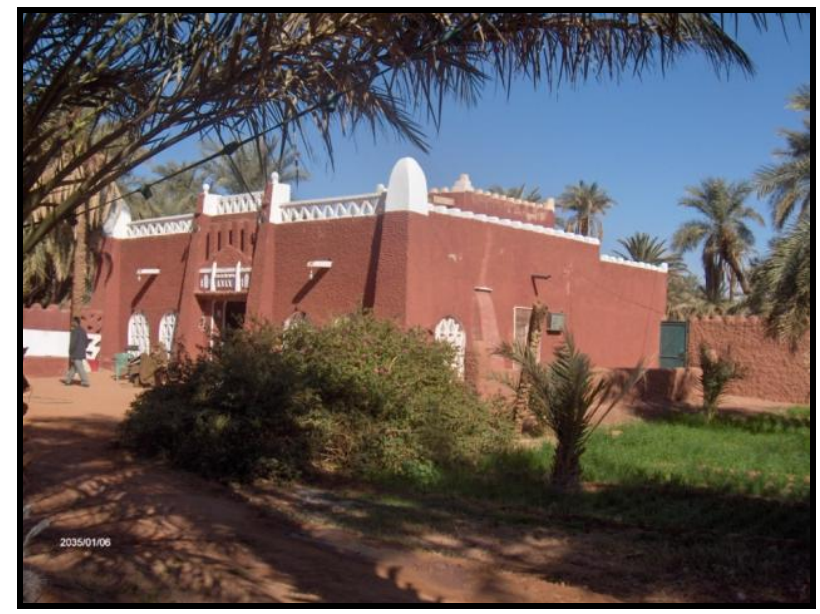

Photo $\mathbf{n}^{\circ}$ 6. Nouvelle résidence construite dans les jardins de l'oasis de Timimoun (propriétaire d'Alger)

\section{Conclusion}

L'intégration des nouveaux habitants dans les villes sahariennes du Sud-ouest algérien s'est faite par un processus de mobilité qui a produit quatre formes distinctives d'espace.

La première forme des nouveaux espaces urbanisés a été érigée par la colonisation où des nouveaux villages ont fait face aux ksour. Structurés d'une manière technico-militaire, ces

\footnotetext{
${ }^{19}$ Nous en avons recensé deux en 2008.
} 
espaces sont ouverts vers l'extérieur et constituent l'espace des étrangers. Des zones ont été réservées à la population algériennes (indigènes) intégrés dans le processus économique et politique colonial (méharistes et ouvriers) puisque peu d'européens s'y sont installés mis à part à Bechar et Kenadsa où la discontinuité entre les deux espaces était bien marquée.

La deuxième forme a été constituée par des quartiers autoproduits qui se sont formés suite à la sédentarisation des nomades qui transhumaient dans la région. Ce phénomène qui a commencé avec la colonisation s'est propagé dans les deux décennies qui ont suivi l'indépendance. Il faut rappeler que le changement des modes économiques a anticipé cette mutation. C'est ainsi que de quartiers formés selon des logiques d'appartenance tribale ont été produits dans quelques villes. Dans la même logique, se rajoutent de nouveaux quartiers constitués par des flux migratoires subsahariens, jouant le rôle de supports de la migration transnationale.

Quant à la troisième forme des nouveaux espaces urbains, elle est le produit d'une forte intervention de l'Etat Algérien dans la production du logement. Ceci se distingue par la multiplication des programmes affectés selon les catégories sociales. Ces logements ont été destinés aussi bien pour reloger la population locale que pour les nouveaux habitants. En effet, les habitants des anciens noyaux en détresse à savoir les ksouriens ont fait l'objet de plusieurs opérations de relogement dans les nouveaux espaces urbanisés suite à la détérioration de ce type d'habitat par les intempéries. D'un autre coté, ces espaces sont convoités par la population originaire des oasis environnantes, dans une stratégie d'accéder à la ville. Leurs intégration à la ville se fait par le logement en s'inscrivant dans les nouveaux programmes d'aide au logement (Logements socio-participatifs, lotissements et l'habitat rural, logements évolutifs). Ces espaces sont aussi le résultat d'une forte mobilité professionnelle conjuguée aux politiques menées par l'Etat en matière d'aménagement de l'espace national et d'intégration des espaces sahariens. Des cadres et des commerçants nordistes sont venus s'installer au sud pour accompagner cette dynamique urbaine. Le logement des fonctionnaires occupe une part importante dans ces nouveaux espaces urbanisés dans l'habitat collectif d'un côté où des cités entières leur ont été réservées et dans l'habitat individuel d'un autre côté, par le biais des coopératives immobilières dont une grande partie n'est pas achevée, révélant une stratégie résidentielle temporaire.

Enfin, la dernière forme est celle du mitage des palmeraies des oasis les plus urbanisées et la reconquête des ksour par une population originaire des métropoles du nord du pays à travers l'acquisition d'une résidence secondaire. Bien que ce phénomène reste limité en nombre et à une échelle nationale par rapport à ce qui se passe dans les pays voisins notamment au Maroc, il est à signaler que le nombre des actions n'a cessé d'évoluer d'une année à l'autre.

\section{Références bibliographiques :}

BAALA Z., BENSALEM K., 2009 : Difficultés d'intégration d'un quartier spontané dans la ville d'Adrar : haï Beni Ouskout, Mémoire d'ingénieur en géographie, Université d'Oran. Mémoire en langue arabe. $129 \mathrm{p}$.

BENDJELID A.- BRULE J.C., FONTAINE J., 2004 : Aménageur et aménagés en Algérie, L'Harmattan, Paris.

BENDJELID A., 1997 : «Anthropologie d'un nouvel espace habité : enjeux fonciers et spatialités des classes moyennes à Oran », Insaniyat n 2, CRASC, Oran, p.5 à p.26. 
BENSAAD A., (dir.), 2009: le Maghreb à l'épreuve des migrations subsahariennes, immigration sur émigrant, Karthala, Paris.

BISSON J., 1960 : «Evolution récente des oasis du Gourara (1952-1959) », extrait de travaux de l'Institut de Recherches Sahariennes $1^{\text {er }}$ et $2^{\text {ème }}$ semestre 1960.

BISSON J., 1986 : « Ksour du Gourara et du Tafilelt, de l'ouverture de la société oasienne à la fermeture de la maison », E.CNRS, In Annuaire de l'Afrique du Nord, Tome XXV.

BISSON J., 2003 : Le Sahara : mythes et réalités d'un désert convoité, Paris, L'Harmattan, 479p.

BLIN L., 1990 : L'Algérie du Sahara au Sahel, Paris, L'Harmattan, 502p.

BOESEN E. ET MARFAING L., (dir.), 2007 : les nouveaux urbains dans l'espace SaharaSahel, un cosmopolitisme par le bas, Karthala, Paris.

CAPOT-REY R., 1953 : La Sahara français, Presses Universitaires (collection Pays outre Mer), $464 \mathrm{p}$.

COTE M, (dir.), 2005 : la ville et le désert, le Bas Sahara algérien, Aix-en-Provence, Karthala, IREMAM, Paris, 306p.

COTE M., 1998 : «Dynamique urbaine au Sahara », Insaniyat $\mathrm{N}^{\circ} 05,1998$, CRASC d'Oran, pp.85-92.

FOUNDOU G.A., 2005, Etude des nouvelles extensions de la ville d'Adrar, mémoire d'ingénieur en Géographie, Université d'Oran, Mémoire en langue arabe.143p.

KOUZMINE Y., FONTAINE J., YOUSFI B., OTMANE T., 2009 : «Etapes de la structuration d'un désert : l'espace saharien algérien entre convoitises économiques, projets politiques et aménagement du territoire », Annales de géographie n 670 (6/2009) Varia, Décembre 2009 (pages 91 à 117).

KURZAC-SOUALI A.C., 2009, «Les médinas marocaines : une requalification sélective », Les Cahiers d'EMAM, 17 | 2009, 117-120.

MAAROUF N., 1980 : Lecture de l'espace oasien, Paris, Sindbad, 286p.

SOUAMI T., 2004 : Aménageurs de villes et territoires d'habitants, un siècle dans le sud algérien, Paris, L'Harmattan, 420p.

TLIDJI N., ABDELKADER A., 2004 : Les quartiers périphériques : planification, réalité et relation avec la ville mère: cas de la zone bleu à Bechar, Mémoire d'ingénieur en géographie, Université d'Oran. Mémoire en langue arabe.107p.

TRACHE S.M., 2011 : « Adrar, des ksour à la grande ville », Insaniyat, 51-52, CRASC, Oran, pp.139-155.

Troin J.F., Bisson J., Bisson V., Brûlé J.C., Escallier R., Fontaine J., - Signoles P., (dir.), 2006 : Le Grand Maghreb, Armand Colin, Paris.

YOUSFI B., 2011 : «La ville d'Adrar dans un nouveau contexte urbain : vers une nouvelle réorganisation de l'espace régional des territoires sahariens du sud-ouest de l'Algérie », Insaniyat, $\mathrm{n}^{\circ}$ 51-52, CRASC, Oran, pp.27-50. En langue arabe

YOUSFI B., 2012, Dynamiques urbaines, mobilités et transports dans le Sud-ouest algérien (Wilayas d'Adrar et de Bechar), Thèse de doctorat en Géographie et en Aménagement du Territoire, Université d'Oran et Université de Franche-Comté, 444p.

YOUSFI B., 2013, Mutation de 1'habitat. Dans le Sud-ouest algérien, In Le Sociographe $N^{\circ} 44$ «Nébuleuses du logement social en France et Afrique du Nord », p.p. 45-52.

YOUSFI B., 2014, Dynamiques urbaines des villes sahariennes, petites et moyennes, du Sud-ouest algérien, In Cahiers de démographie locale 2012 «dynamiques des populations locales », Néothèque, coll.pp. 251-281. 